\title{
Asymptotic Delay Analysis for Cross-Layer Delay-Based Routing in Ad Hoc Networks
}

\author{
Philippe Jacquet, ${ }^{1,2}$ Amina Meraihi Naimi, ${ }^{2}$ and Georgios Rodolakis ${ }^{1,2}$ \\ ${ }^{1}$ Ecole Polytechnique, 91128 Palaiseau Cedex, France \\ ${ }^{2}$ Institut National de Recherche en Informatique et en Automatique, Unité de recherche de Rocquencourt, \\ 78153 Le Chesnay Cedex, France
}

Received 21 December 2006; Revised 20 April 2007; Accepted 14 May 2007

Recommended by Haohong Wang

\begin{abstract}
This paper addresses the problem of the evaluation of the delay distribution via analytical means in IEEE 802.11 wireless ad hoc networks. We show that the asymptotic delay distribution can be expressed as a power law. Based on the latter result, we present a cross-layer delay estimation protocol and we derive new delay-distribution-based routing algorithms, which are well adapted to the QoS requirements of real-time multimedia applications. In fact, multimedia services are not sensitive to average delays, but rather to the asymptotic delay distributions. Indeed, video streaming applications drop frames when they are received beyond a delay threshold, determined by the buffer size. Although delay-distribution-based routing is an NP-hard problem, we show that it can be solved in polynomial time when the delay threshold is large, because of the asymptotic power law distribution of the link delays.
\end{abstract}

Copyright ( $) 2007$ Philippe Jacquet et al. This is an open access article distributed under the Creative Commons Attribution License, which permits unrestricted use, distribution, and reproduction in any medium, provided the original work is properly cited.

\section{INTRODUCTION}

With the emergence of real-time applications in wireless networks, delay guarantees are increasingly required. In order to provide support for delay sensitive traffic in such networks, an accurate evaluation of the delay is a necessary first step. Moreover, in the case of mobile ad hoc networks, a detailed understanding of the impact of both the underlying MAC and routing protocols on the delay characteristics is crucial, since the traffic routes consist of several wireless hops. In this paper, we address the problem of the analytical evaluation of the delay distribution in a multihop wireless network. We consider a wireless network with IEEE 802.11 MAC protocol [1] under the optimized link state routing (OLSR) protocol [2]. The routing protocol is a table-driven protocol that operates under periodic broadcast control packets. IEEE 802.11 is the most popular MAC protocol in wireless LANs and mobile ad hoc networks. The main channel access mechanism is the distributed coordination function (DCF), which is a carrier sense multiple access with collision avoidance (CSMA/CA) scheme. DCF was designed initially for asynchronous traffic and since it is a random access protocol it does not provide any guarantee for delay sensitive applications.
The performance of the 802.11 protocol in single-hop wireless networks has been addressed in the past in several papers. A simple analytical model of the 802.11 DCF access mechanism was introduced in [3] and was used to analyze the saturation throughput performance. The MAC layer service time was studied in [4], by expanding the previous model. The delay in both saturated and unsaturated networks was also studied in [5], where each node was modelled as a discrete time queue. Multihop delay was studied in [6], in the context of mesh networks.

In this work, we evaluate the 802.11 protocol performance in terms of delay in the context of wireless ad hoc networks. We focus on the asymptotic properties of the delay and we obtain both one hop and multihop analytical delay estimates. We show that the asymptotic delay distribution can be expressed as a power law. Based on the latter result, we present a cross-layer delay estimation protocol and we derive new delay-distribution-based routing algorithms, which are well adapted to the QoS requirements of real-time multimedia applications.

We take the slotted time approach of [3]. We denote by $W$ the end-to-end delivery delay of a packet. We analyze the delay distribution $P(W>T)$ and we show that in the case that 
$T$ is large (i.e., several times the average delay) the probability $P(W>T)$ decays as a power law, namely in $O\left(T^{-a}\right)$, where $a$ is a constant. In order to simplify the formula derivations, we perform the analysis under certain modelling assumptions. For instance, we use an M/G/1 queueing model for the nodes (a detailed description is provided in the methodology section), which cannot be considered a priori entirely realistic. However, we have verified via simulations that our model is pertinent and can accurately predict the shape of the node delay distributions in the domain of interest. Furthermore, the assumptions we make are not fundamental for our results, therefore we comment on plausible model generalizations whenever possible.

Based on the analysis, we present a cross-layer framework to evaluate the delay distribution $P(W>T)$ for any large $T$ and use it in order to find routes that satisfy given delay requirements. A delay oriented quality of service for a connection is generally expressed via a maximum acceptable delay $T$ and a maximum over-delay ratio $\epsilon$, specified by the application, requiring that during the connection the constraint $P(W>T)<\epsilon$ is verified. In general finding the optimal route that minimizes an over-delay ratio is NP-hard [7]. Nevertheless, the fact that the delay distribution at every node router is in power law allows us to specify a polynomial approximation algorithm with an error factor of $1+O\left(T^{-1}\right)$.

The rest of the paper is organized as follows. In Section 2, we present an overview of the IEEE 802.11 DCF mode. In Section 3 we introduce the general model framework and we analyze the one hop delay. The multihop delay distribution is derived in Section 4. In Section 5 we describe a crosslayer delay estimation protocol based on OLSR. In Section 6 we present some simulation results. Finally, in Section 7, we indicate how the previous analysis can be used in delaydistribution-based routing.

\section{802.11 DISTRIBUTED COORDINATION FUNCTION OVERVIEW}

The distributed coordination function (DCF) is the fundamental access method used in the IEEE 802.11 MAC protocol [1]. It is based on the carrier sense multiple access with collision avoidance (CSMA/CA) mechanism, which is designed to reduce the collisions due to multiple sources transmitting simultaneously on a shared channel. In the CSMA/CA protocol, a station transmits only if the medium is idle. The medium is considered as idle if it is sensed to be idle for a duration greater than the distributed inter-frame space (DIFS). If the medium is sensed as busy, the transmission is deferred until the end of the ongoing transmission. When the medium becomes idle after a busy period, the node does not transmit immediately, because multiple stations could have been waiting for the end of the transmission and may attempt to access the channel again at the same time. Therefore, the node starts a random wait by initializing its backoff timer. The backoff timer is randomly selected in an interval called the contention window and has the granularity of one slot. Every time the channel is sensed to be idle, the backoff counter is decre- mented. When the counter reaches zero, the node can start its transmission. If the channel is sensed as busy during the backoff procedure, the counter is frozen and then resumed when the channel becomes idle for a DIFS again. In spite of that, collisions can still occur. In order to reduce the probability of further collisions, the contention window is doubled after each collision to increase the random waiting time. The exponential backoff function is discussed in a more detailed manner in Section 3.2. To make sure that the transmitted frame has reached its destination, an acknowledgment frame is generated from the destination to the source.

The above carrier sense is called physical carrier sense because it is performed at the air interface. A virtual carrier sense is also possible in the DCF mode to resolve the problem of the hidden terminal. This problem occurs when two nodes that are not within hearing distance of each other create collisions at a third terminal that receives the transmission from both. The virtual carrier sense is performed at the MAC sub-layer. The channel is reserved before each transmission, so instead of transmitting the data frame after sensing that the channel is idle, the station sends an RTS (request to send) frame to the destination. The receiver replies by a CTS (clear to send) frame after which data transfer can start. However, the use of RTS/CTS frames imposes additional delay and bandwidth overhead. Therefore the RTS/CTS mechanism is recommended only for big packets.

\section{ONE-HOP DELAY DISTRIBUTION ANALYSIS}

\subsection{Methodology overview}

A wireless node can be seen as a buffer filled by incoming messages and with a single server that performs the CSMA/CA multiple access protocol. We model this system as an $\mathrm{M} / \mathrm{G} / 1$ queue, that is, we assume

(1) the input packet flow in the buffer is Poisson of rate $\lambda$;

(2) service delays are independent.

In fact, the M/G/1 hypothesis is just a matter of simplifying approach. Since we are going to deal with heavy tailed distribution of service times, the consequence on queueing time distribution can be generalized to a much larger class of queueing models. For example, it is not necessary to assume independence between service times or to restrict to Poisson input in order to derive a power law queueing distribution, but in this case the coefficients change (see [8] for the case of a GI/GI/1 queue). Nonetheless, as we verify later in the simulations section, the M/G/1 hypothesis leads to satisfactory results.

\subsection{Service delay determination}

The IEEE 802.11 CSMA/CA protocol uses a rotating backoff where the nodes have to wait a random number of idle slots between transmission attempts. Let $\mathbf{C}$ be the random variable that expresses the number of busy slots between two 
consecutive idle slots. Let $p(L)$ be the probability of collision that is experienced by packets, when the packet length is $L$ (i.e., the physical transfer time of the packet in the channel, expressed in slots, which is proportional to its size in bits). The longer the packet is, the more likely it is to collide. We take the following assumptions.

(1) Durations between successive idle slots are independent and identically distributed.

(2) Collision events on successive transmissions are independent.

According to the CSMA protocol, the backoff counter is selected in an initial interval $\left\{1, \ldots, W_{\min }\right\}$. If a collision occurs the nodes select a new backoff number in an enlarged interval $\left\{1, \ldots, 2 W_{\min }\right\}$. The backoff interval length is multiplied by two after each collision. The backoff interval length is reset to $W_{\min }$ for the next packet. In practice there is a maximum number of retries after which the packet is discarded in case of permanent failure. The default maximum retry is 7 and can lead to a delay on the order of seconds. Since this delay is larger than the maximum acceptable delay we think of regarding connection QoS, it does not practically matter to set the maximum number of retries to infinity.

Let $C(z)$ be the probability generating function $\sum_{n} P(\mathbf{C}=n) z^{n+1}$, quantity $C$ being expressed in slot duration. This generating function corresponds to the time needed for a backoff counter decrease, expressed by the random variable $C+1$ (we add one slot to the quantity $C$ for the decrease to be taken into account). Identity $C(z)=z$ would mean that $\mathbf{C}=0$ always, that is, the channel is permanently sensed idle (note that in this case one slot is still needed for the counter decrease).

Let $\beta(z, L, p, k)$ be the probability generating function of the service delay when the packet length is $L$, the collision probability is $p$, and the initial backoff interval is $k$. The service delay of a packet corresponds to the time elapsed since it was extracted from the buffer until it is transmitted successfully. Therefore, it takes into account retransmissions due to collisions and it includes the time needed to access to the channel (corresponding to the rotating backoff decrementation) plus the fixed packet transmission length.

We will express all these quantities using generating functions, starting from the time needed to access the channel, or equivalently the backoff counter decrease. As discussed earlier, each backoff decrease is expressed by the random variable $C+1$, with generating function $C(z)$. If the backoff counter is $i$, the total time to access the channel is the time needed for $i$ counter decreases, or the sum of $i$ times the random variable $C+1$. From the independence assumption it comes that in this case the channel access time can be expressed by generating function $C(z)^{i}$. Since the initial backoff window is $k$, and the backoff counter value is selected uniformly at random in the interval $\{1, \ldots, k\}$ (we also take here into account the DIFS interval), the generating function of the total channel access time can be written as $(1 / k) \sum_{i=1, \ldots, k} C(z)^{i}$, which results from the previous discussion by taking either possible value for $i$ with probability $1 / k$. Once the channel is accessed, the time needed to transmit the packet is fixed and equal to $L,{ }^{1}$ therefore it can be expressed by generating function $z^{L}$. Hence the service time when no collision occurs comes from adding the previous two quantities, or equivalently the corresponding generating function is equal to the product of the above generating functions, that is,

$$
\frac{z^{L}}{k} \sum_{i=1, \ldots, k} C(z)^{i}=\frac{C(z)^{k+1}-C(z)}{C(z)-1} \frac{z^{L}}{k} .
$$

In order to account for packet collisions, we obtain the following recursion:

$$
\beta(z, L, p, k)=\frac{C(z)^{k+1}-C(z)}{C(z)-1} \frac{z^{L}}{k} \times(1-p+p \beta(z, L, p, 2 k)) .
$$

In case there is no collision (with probability $1-p$ ), the service delay corresponds to our previous calculations. The term $\beta(z, L, p, 2 k)$ is obtained from the case where there is a collision (with probability $p$ ), hence the procedure is repeated after doubling the backoff interval and this results in an additional service delay term.

The service delay probability generating function is

$$
\beta(z)=E\left[\beta\left(z, L, p(L), C W_{\min }\right)\right],
$$

which is obtained by averaging on packet length $L$ and collision probabilities $p(L)$.

Figure 1 shows the 200 first coefficients of $\beta(z)$ when $C(z)=0.8 z+0.2 z^{4}, L=4$ and $p=0.3$. In this theoretical example, the packet transferring time is 4 slots, and each decrementation of the backoff counter takes one slot with probability $80 \%$ and 4 slots with probability $20 \%$ (which means that the channel is busy with a packet transmission). The coefficients in this figure were obtained from numerical calculations using Maple, by iterating the recursive equation (2).

Remark 1. In case the RTS/CTS mechanism is used, the recursive equation (2) becomes

$$
\begin{aligned}
\beta(z, k)= & \frac{1}{k} \frac{C(z)^{k+1}-C(z)}{1-C(z)} \\
& \times\left(\left(1-p_{1}-p_{2}\right) z^{r+L}+\left(p_{1} z^{r}+p_{2} z^{r+L}\right) \beta(z, 2 k)\right),
\end{aligned}
$$

where $r$ is the RTS transmission time, $p_{1}$ and $p_{2}$ are the collision probabilities on RTS and data frames, respectively.

Note that in general $p_{1}>p_{2}$, due to the channel reservation.

The modified recursive equation consists again of a backoff decrementation term $(1 / k)\left(\left(C(z)^{k+1}-C(z)\right) /(1-C(z))\right)$, and there are two cases for the additional delay:

(i) if there is no collision (with probability $1-p_{1}-p_{2}$ ) the delay is equal to the RTS plus the data frame transmission time (term $z^{r+L}$ );

\footnotetext{
${ }^{1} L$ can be adjusted to include AckTimeouts too.
} 


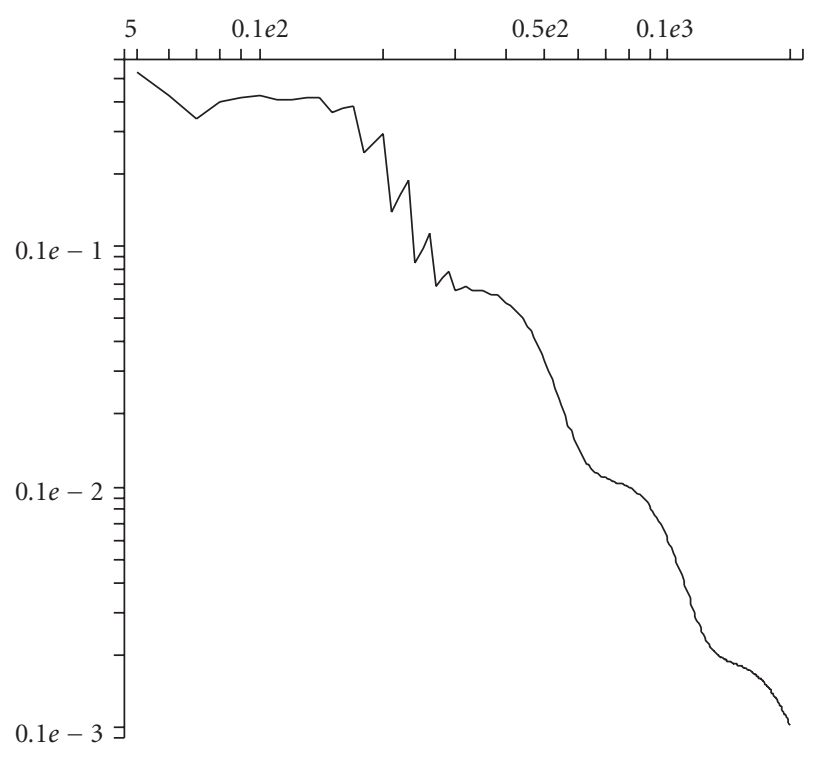

Figure 1: Coefficients of $\beta(z)$.

(ii) in case there is a collision on either an RTS frame (term $p_{1} z^{r}$ ) or a data frame (term $p_{2} z^{r+L}$ ) the procedure is repeated.

\subsection{Delays including queueing}

In order to compute the delay experienced by packets in the buffer, we take the formula for slotted M/G/1 for the queue delay probability generating function $q(z)$

$$
q(z)=\exp \left((\beta(z)-1) \frac{\lambda}{2}\right) \frac{\left(1-\lambda \beta^{\prime}(1)\right)(1-z)}{1-z \exp (-(\beta(z)-1) \lambda)} .
$$

This needs the provision that $\beta^{\prime}(1)$ exists. We will see that this implies that $p<1 / 2$. Similarly, for the existence of the $k$ th moment of service time we need that $p<2^{-k}$. If $\lambda \ll 1$ then we can replace (5) by

$$
q(z) \approx \frac{\left(1-\lambda \beta^{\prime}(1)\right)}{1-(z /(1-z))(1-\beta(z)) \lambda} .
$$

The generating function of the overall delay, so called one hop delay (queueing + service), of a packet of length $L$ with collision probability $p, w(z, L, p)$ satisfies the identity

$$
w(z, L, p)=q(z) \beta\left(z, L, p, W_{\min }\right) .
$$

Figure 2 shows the coefficients of $w(z)$ for $\lambda=0.02$. Notice that $\beta^{\prime}(1)=22.939 \ldots$

\subsection{Asymptotic analysis}

We denote by $S$ the service time and $W$ the overall delay in a router. In this section we derive asymptotic estimates for the distributions of the above quantities by applying FlajoletOdlyzko theorems [9]. The proofs are given in the appendix.

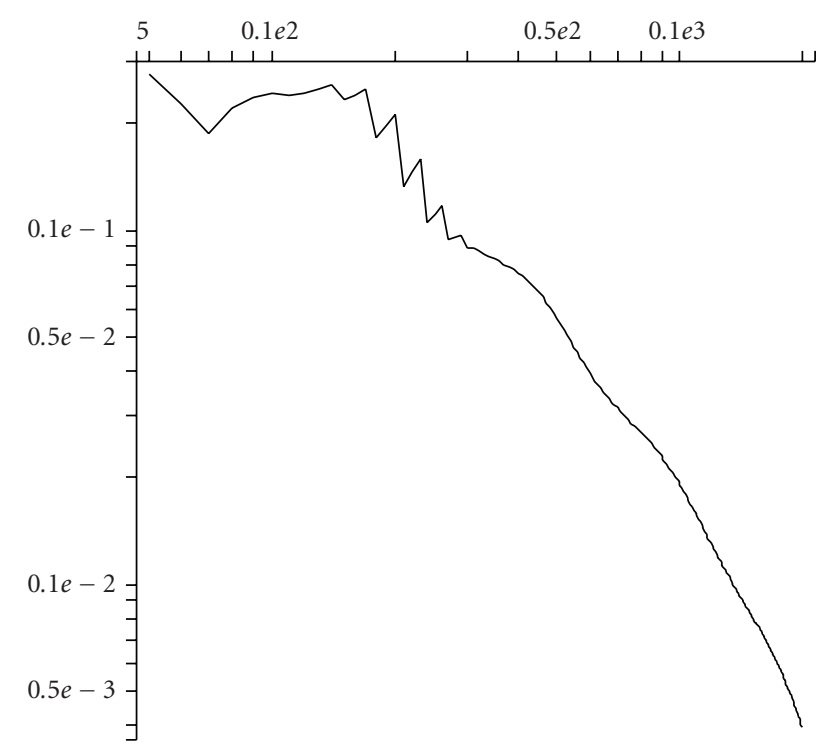

Figure 2: Coefficients of $w(z)$.

Theorem 1. The expansion for $z$ around 1 holds:

$$
\begin{aligned}
\beta(z, L, p, k)= & 1+(1-z) v(1-z) \\
& +\left(k C^{\prime}(1)(1-z)\right)^{B} \alpha(\log (1-z)) \\
& +O\left((1-z)^{B+1}\right)
\end{aligned}
$$

where $v(x)$ is a polynomial, $B=-\log _{2} p$ assuming that $B$ is not integer, and $\alpha(x)$ is a periodic function of period $\log 2$ with small fluctuation.

Theorem 2. The probability that the service time is greater than $T$, for $T$ large is

$$
P(S>T)=\left(W_{\min } C^{\prime}(1)\right)^{B} \alpha^{*}(\log T) T^{-B}+O\left(T^{-B-1}\right),
$$

where $\alpha^{*}(x)$ is also a periodic function of period $\log 2$ with small fluctuation.

Theorem 3. The expansion for $z$ around 1 holds:

$$
\begin{aligned}
w(z)= & 1+(1-z) u(1-z) \\
& +\frac{\lambda\left(W_{\min } C^{\prime}(1)\right)^{B}}{1-\lambda \beta^{\prime}(1)} \alpha(\log (1-z))(1-z)^{B-1} \\
& +O\left((1-z)^{B}\right),
\end{aligned}
$$

where $u(x)$ is an analytic function.

Theorem 4. The probability that the delay in a router is greater than $T$, for $T$ large is

$$
P(W>T)=\frac{\lambda\left(W_{\min } C^{\prime}(1)\right)^{B}}{1-\lambda \beta^{\prime}(1)} \alpha^{*}(\log T) T^{1-B}+O\left(T^{-B}\right) .
$$


Notice that the delay distribution tail decays in power law. As a corollary it turns out that the existence of the $k$ th moment of the delay needs $p<2^{-k-1}$. Also, to obtain the asymptotic delay distribution estimate, only the average of the channel occupancy distribution $C^{\prime}(1)$ is required, rather than the distribution $C(z)$.

Remark 2. Similarly, in case the RTS/CTS mechanism is used, we have the expansion $\beta(z, k)=1+z f(z)+c_{2}((1-$ $z) k)^{B}+O\left((1-z)^{B+1}\right)$ with $B=-\log \max _{i=1,2}\left\{p_{i}\right\}=-\log p_{1}$ and $f(z)$ an analytical function. This implies that both service time and delay distributions are asymptotically power laws.

\section{MULTIHOP DELAY ANALYSIS}

\subsection{General case: correlated waiting times in queues}

We now compute the end-to-end (multihop) delay distribution for any given route in the network, based on the one hop delay analysis discussed previously. For this purpose, we assume that when travelling on its route, the delay experienced by a packet on a router is independent of the delay experienced on other routers. This assumption makes the problem easier to handle mathematically. However, it is not a fundamental assumption for our result since it is known that the sum of several random variables in power law is still in power law whatever the dependence assumptions between them. The power law in the resulting distribution function will be the maximum of the respective power laws of the variables, except that the factor in front of it will depend on the dependence assumptions. To see this, consider $n$ random variables $X_{1}, \ldots, X_{n}$, such that $P\left(X_{i}>T\right) \propto T^{-B_{i}}$ for all $1 \leq i \leq n$. We will show that $X_{1}+\cdots+X_{n}$ is also in power law, and the value on the power is always the same regardless of any correlation between the random variables. Moreover, we will determine appropriate lower and upper bounds on the coefficients.

First of all an easy lower bound: we have $P\left(\sum_{i} X_{i}>T\right) \geq$ $\max _{i}\left\{P\left(X_{i}>T\right)\right\}$.

Second, we can obtain an upper power law bound since

(1) $\sum_{i} X_{i} \leq n \max _{i}\left\{X_{i}\right\}$

(2) $P\left(\max _{i}\left\{X_{i}\right\}>T\right)=P\left(\exists i X_{i}>T\right) \leq \sum_{i} P\left(X_{i}>T\right)$.

Therefore $P\left(\sum_{i} X_{i}>T\right) \leq \sum_{i} P\left(X_{i}>T / n\right) \propto \sum_{i} n^{B_{i}} T^{-B_{i}}$, independently of any correlation between the variables, and derive appropriate upper bound delay routing in power law if the $X_{i}$ 's are the waiting delays in routers. Notice that the lower bound and upper bounds are asymptotically within a factor $n^{B}$ of each other, with $B=\min _{i}\left\{B_{i}\right\}$.

\subsection{Independence assumption}

Assuming independence from now on, we can work on the exact asymptotic value instead of the bounds obtained in the general case, keeping in mind that these bounds are within a factor $n^{B}$ of the exact value, where $n$ is the route length and $B=\min _{i}\left\{B_{i}\right\}$.

Taking fully the independence assumption, when there are $n$ routers in the route from the source to the destination, the probability generating function of the end-to-end delay is equal to the product $\prod_{i \in \text { route }} w_{i}(z)$ where $w_{i}(z)$ is the probability generating function of the delay at router number $i$ and route is a set of router indices.

Still, according to Flajolet-Odlyzko results [9], if each $w_{i}(z)$ is of the form $1+(z-1) g_{i}(z)+c_{i}(z-1)^{B_{i}-1}+O\left((z-1)^{B_{i}}\right)$, where $c_{i}$ is a constant, then the leading term of $P(W$ (route) $>$ $T)$ is $\sum_{i \in \text { route }} c_{i}^{*} T^{1-B_{i}}$, with $c_{i}^{*}=c_{i} / \Gamma(2-B)$. Keeping only leading terms

$$
P(W(\text { route })>T) \approx c(\text { route }) T^{1-B(\text { route })},
$$

where $B($ route $)=\min B_{i}$ and $c($ route $)=\sum_{B_{j}=B} c_{j}^{*}$.

An unexpected consequence of the above is that a good choice for the route should not be the shortest path in number of hops. In the shortest path the lap between two consecutive routers may be too large, leading to too large collision rates and therefore a too low value of $B$ (route). If we take shorter hops between routers, then we will reduce the collision rate and get a larger value of $B$ (route). Of course this would be done in the detriment of a larger number of hops and a larger value of $c$ (route). But, since in $c$ (route) $T^{1-B \text { (route) }}$ parameter $T$ is supposed to be large, the reduction of $T^{1-B \text { (route) }}$ would prevail in most cases on the $c$ (route) increase.

Interestingly enough, increasing the number of hops and $c$ (route) will in most cases increase the average end-to-end delay. Therefore we have the paradoxical case where increasing the average delay actually decreases the over-delay loss ratio. This is due to the fact that we expect the average delay to be much lower than the maximum acceptable delay $T$. Consequently, routing with respect to average delay as it is done in [10] may conflict with the minimization of the over-delay ratio. Conversely, the optimal route may be too long since it may have too short hops. In this case the connection may waste too many resources. Instead of choosing the route that minimizes $P(W>T)$ it is probably wiser to seek the shortest route that satisfies the requirement $P(W>T) \leq \epsilon$.

\section{CROSS-LAYER DELAY ESTIMATION PROTOCOL}

In this section, we present a delay estimation protocol, used to obtain estimates of the delay distributions for all routes in the network in a proactive way. In Section 7 we show how this information can be used to optimize route computation. In fact, we propose an extension to the OLSR routing protocol to support delay estimation for any given route in a mobile ad hoc network.

Note that our delay distribution analysis holds regardless of the mobility of nodes. In fact, the end-to-end delay distribution analysis concerns a given route. Consequently, any changes in the path from the source to the destination result in a new power law for the end-to-end delay distribution. Our proposed delay estimation protocol is designed to support mobility in an ad hoc network. It is proactive and predictive, in the sense that, using the previously presented analytical model, it is able to estimate delays on links and routes even if no data packets have been transmitted yet. Consequently, we permanently have a prediction of the delay 


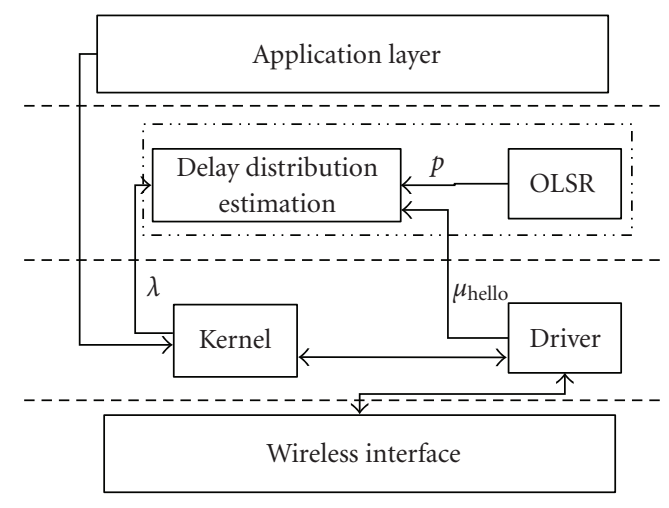

FIGURE 3: Delay estimation protocol framework.

distribution over all routes in the network, which can be used by a delay based routing protocol.

As mentioned previously, the single-hop delay distribution estimate is based on the knowledge of the collision probability and the average of the channel occupancy distribution $C$, which are basically MAC layer parameters. The multihop delay distribution is based on the knowledge of single hop characteristics along a given route, which clearly concerns the functioning of the routing protocol. Therefore, the extended protocol needs to interact with the MAC layer. In Figure 3, we depict the protocol framework.

\subsection{Average of channel occupancy distribution $C$}

The channel occupancy information concerns the internal functioning of wireless cards and is not actually known. However, the card acknowledges successful frame transmissions by sending special interrupts to the driver. This allows to measure the service time of transmitted packets. Knowing the service time, it is possible to deduce the access time in case of broadcast packets such as OLSR Hello messages (since they are not retransmitted when a collision occurs). Thus, based on (1), it is possible to derive the mean of the channel occupancy distribution $C$ from the mean of the Hello access time distribution, noted by $\mu_{\text {hello }}$. We have $C^{\prime}(1)=$ $2 \mu_{\text {hello }} /\left(W_{\min }+1\right)$.

\subsection{Collision probability estimation}

The collision probability is estimated by OLSR, since this information is not currently provided by wireless cards. OLSR uses Hellos in order to detect neighbors. A node is a neighbor if and only if the Hello collision rate is below a given threshold. Therefore OLSR has a procedure in the advanced neighbor sensing option that allows to compute the collision rate (link quality level parameter). It uses the Hello message sequence number in order to identify the missing Hellos. However there could be a difficulty in the fact that the collision probability $p(L)$ may depend strongly on packet length $L$. One may expect a dependence of the kind $-\log p(L)=a L+b$ where $a$ and $b$ are scalar coefficients. Since the neighbor has no idea of the size of missing Hellos, the transmitter should advertise the length distribution of its Hellos. Comparing with its received Hello distribution the neighbor would be able to determine the coefficients $a$ and $b$. By default the neighbor assumes $a=0$, that is, all packets have the same collision rate regardless of their length.

\subsection{Advertising link quality}

Multihop delay computation is based on the knowledge of the one hop delays of the route. Thus, each node must inform the entire network of its local information. For this purpose, a link quality advertisement (LQA) message is broadcasted in the network.

In OLSR, broadcast traffic is relayed via multipoint relay (MPR) nodes (nodes elected by their neighbors because they cover their two-hop neighborhood) to consume less resources. In order to save more on control traffic, OLSR offers the possibility for the nodes to advertise a small subset of their neighbor links. The advertised link set can be limited to MPR links, that is, the neighbors that have elected this node as an MPR. In this case the nodes have only a partial knowledge of the network topology, nevertheless, each node knows its own neighbor list and this guarantees that any given node can compute a shortest path to any arbitrary destination.

For our purpose, it is preferable to use the option full OLSR, that is, to advertize the whole neighbor set instead of the MPR selector set. In fact, in order to estimate the multihop delay distribution for all routes in the network, we need the complete network topology given by full OLSR. If the advertized links are limited to the MPR selector set, we have only a partial knowledge of the network topology. In the latter case, the nonadvertised links may offer better possibilities for delay-based routing. Consequently, the complete topology is necessary in order to find an optimal route with respect to the delay. The use of the full-OLSR option introduces an additional overhead due to larger link quality advertisement packets. However, broadcast traffic is still relayed with the optimized MPR-flooding mechanism, which significantly reduces the overhead.

The node advertises for each link $\ell$ the collision rate $p_{\ell}$, and for itself it advertises the global $\lambda$, provided by the kernel as shown in Figure 3 , and the value of $C^{\prime}(1)$, or directly the tuple $\left(p, \lambda\left(W_{\min } C^{\prime}(1)\right)^{B} /\left(1-\lambda \beta^{\prime}(1)\right)\right)$.

\section{SIMULATION RESULTS}

We use the ns-2 [11] simulator to validate our delay modelling. We study various scenarios for different purposes. We compare the analytic service time distribution with the measured service time distribution (obtained by ns-2 simulations). We aim to show that the service time and the sojourn time (in other words the delay including queuing) are in power law. Furthermore, we investigate whether one-hop delays are independent within a route, and finally we show that the end-to-end delay is in power law too. Common simulation parameters are summarized in Table 1. 
TABLe 1: Simulation settings.

\begin{tabular}{l|l}
\hline MAC Parameters & $W_{\min } 32$, slot $20 \mu \mathrm{s}$ \\
Propagation model & Two-ray ground \\
Transmission range & $250 \mathrm{~m}$ \\
Packet size & 1000 bytes \\
Traffic type & Exponential (Poisson) \\
Simulation time & $300 \mathrm{~s}$ \\
Simulation Area & $800 \times 800 \mathrm{~m}^{2}$ \\
Routing protocol & OLSR \\
\hline
\end{tabular}

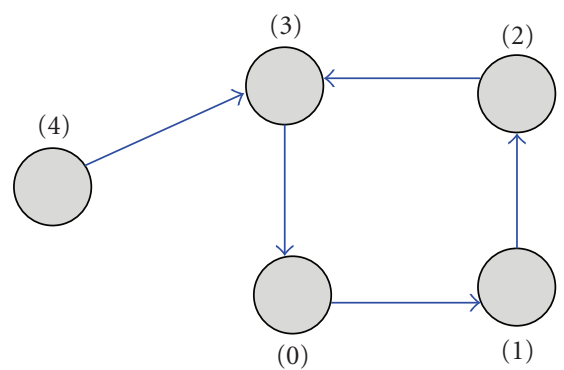

Figure 4: Topology 1.

TABLE 2: Measured parameters.

\begin{tabular}{l|l}
\hline Channel occupancy & $C(z) \simeq 0.82+0.04 z^{15}+0.03 z^{124}+0.1 z^{444}$ \\
Packets per slot & $\lambda=0.00024$ packets $/$ slot $(12$ packets $/ \mathrm{s})$ \\
Collision probability & $p=0.09, B=3.45$ \\
Packet length & $L=229$ slots \\
\hline
\end{tabular}

\section{One-hop delay measures}

In the first scenario, we consider an ad hoc network with 5 nodes as shown in Figure 4. The 802.11 bandwidth is $1 \mathrm{Mb}$. Five exponential flows with $140 \mathrm{kbs}$ data rate are launched between different pairs of nodes (represented by arrows in Figure 4). In order to study the cumulative delay distribution in node 2, we measure the main parameters in this node for the conducted simulation, as presented in Table 2.

Based on these parameters and (2), we compute analytically the service time distribution using Maple, which we draw in Figure 5. The service time distribution measured via ns-2 simulation is shown in Figure 6. To demonstrate that the service time distribution is in power law with $B=-\log _{2}(p)$ (here $B=3.45$ ), as stated in Theorem 2, we draw the equation $Y=\alpha X^{-3.45}$, where $\alpha$ is a constant, and we compare the two plots. Figure 6 shows that, for $T$ large enough, the service time distribution and $Y$ have the same power law exponent.

In the same way, we measure the sojourn time distribution (also called node delay), which we present in Figure 7. We notice that for $T$ between 4000 and 40000 slots (i.e., 80 milliseconds to 800 milliseconds), the node delay is in power law with exponent $1-B=-2.45$, in accordance with Theorem 4.

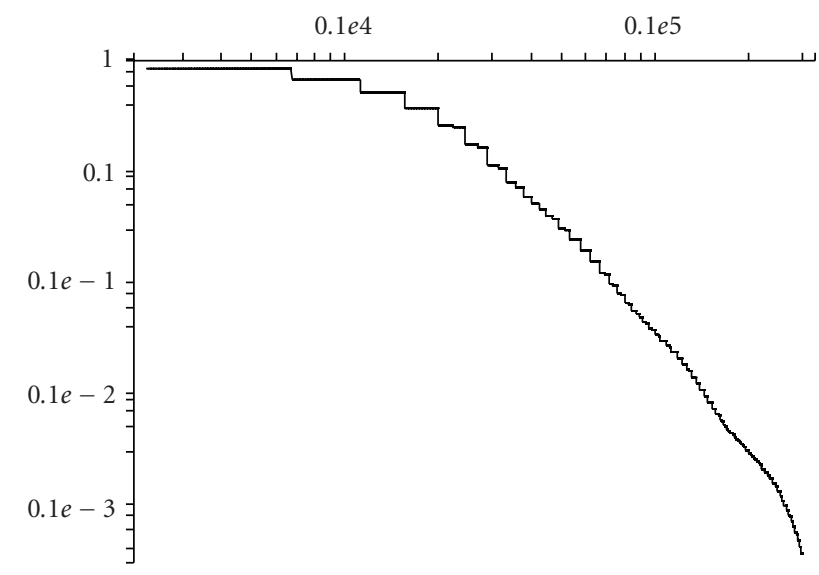

Figure 5: Analytic service time distribution.

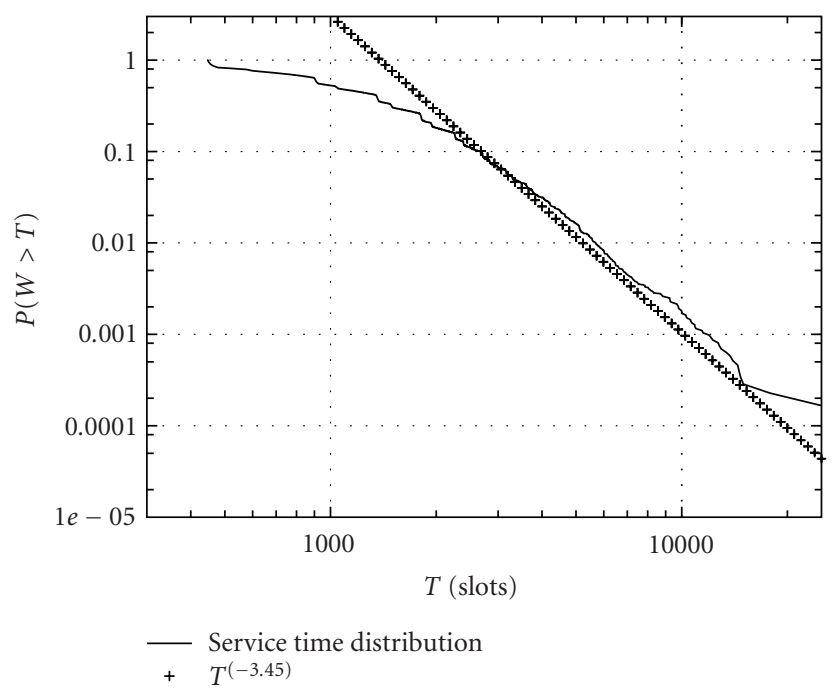

FIgURE 6: Measured service time distribution.

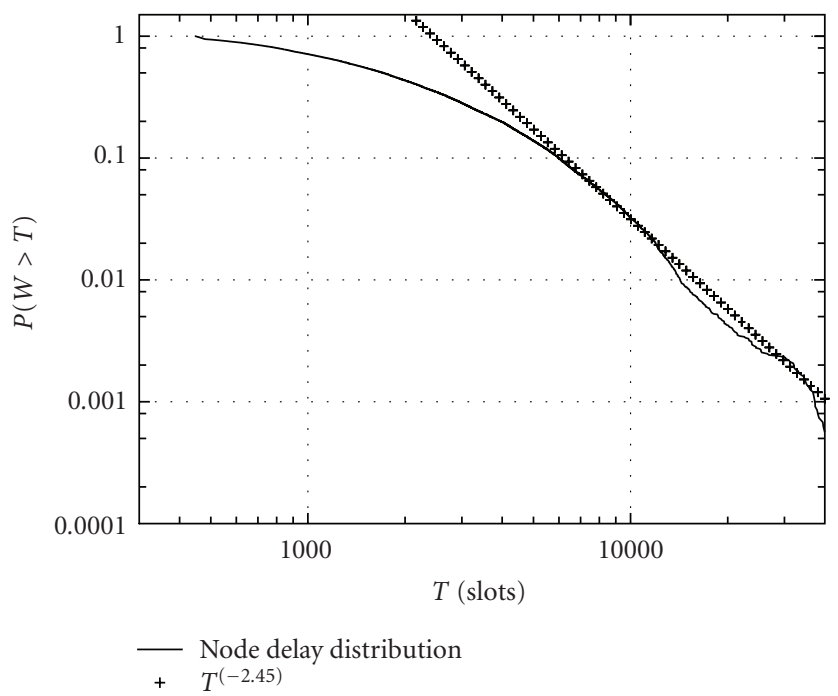

FIgURE 7: Measured node delay distribution. 


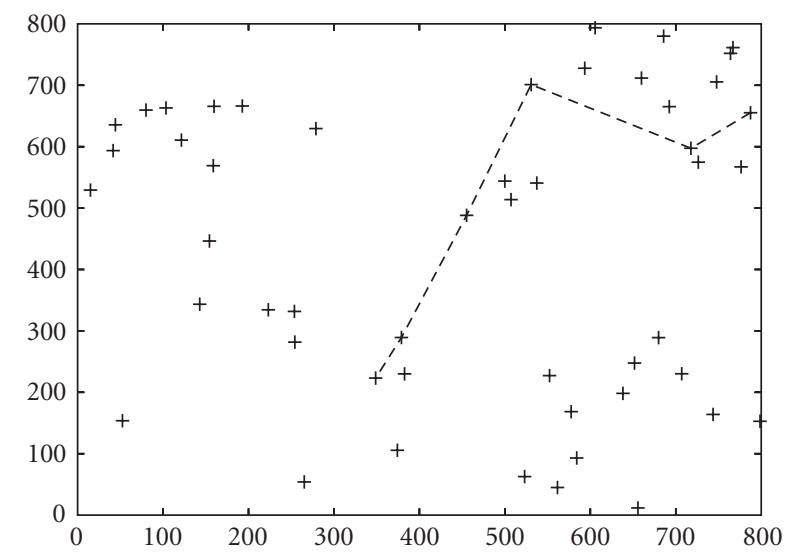

FIgURE 8: Topology 2.

TABle 3: Collision probabilities for each hop of the path.

\begin{tabular}{l|ccccc}
\hline Throughput & \multicolumn{5}{|c}{ Per hop collision probabilities \% } \\
\hline $2 \mathrm{pkt} / \mathrm{s}$ & 0.53 & 0.94 & 0.19 & 1.05 & 1.13 \\
$8 \mathrm{pkt} / \mathrm{s}$ & 1.11 & 2.28 & 0.45 & 5.43 & 5.75 \\
\hline
\end{tabular}

\section{Multihop delay measures}

Secondly, we consider a randomly generated topology of 50 nodes which is depicted in Figure 8. We launch 10 exponential flows in the network and we measure for each the end-toend delay distribution. We run several simulations by varying the throughput from 2 to 8 packets per second. We consider the flow following the five-hop path shown in Figure 8. We measure the end-to-end delay of this flow as well as collision probabilities along the path (for each hop). Table 3 summarizes the obtained probabilities.

Figure 9 compares the measured end-to-end delay distribution with theoretical results for sending rates of 2 and 8 packets per second, respectively. According to the analysis of multihop delay distribution in Section 4, the power law exponent is equal to $1+\log _{2}(p)$ such that $p$ corresponds to the highest collision probability along the path. Referring to Table 3, the highest collision probability on this path is $0.0113(1-B=-5.46)$ and $0.0575(1-B=-3.12)$ for traffic rates of 2 and $8 \mathrm{pkt} / \mathrm{s}$, respectively.

\section{Impact of the packet size on the delay distribution}

Considering the same scenario as previously, we run simulations by varying the packet size from 64 to 1000 bytes as shown in Figure 10. As we notice, for each size, the delay distribution is a power law with slightly different slopes, which correspond to slightly different collision probabilities. For bigger packets there is an increase in the collision probability. For example, for packets of 64 bytes the highest collision probability on the path is $5.5 \%$, while it is equal to $8.2 \%$ for packets of 1000 bytes. In addition, we notice

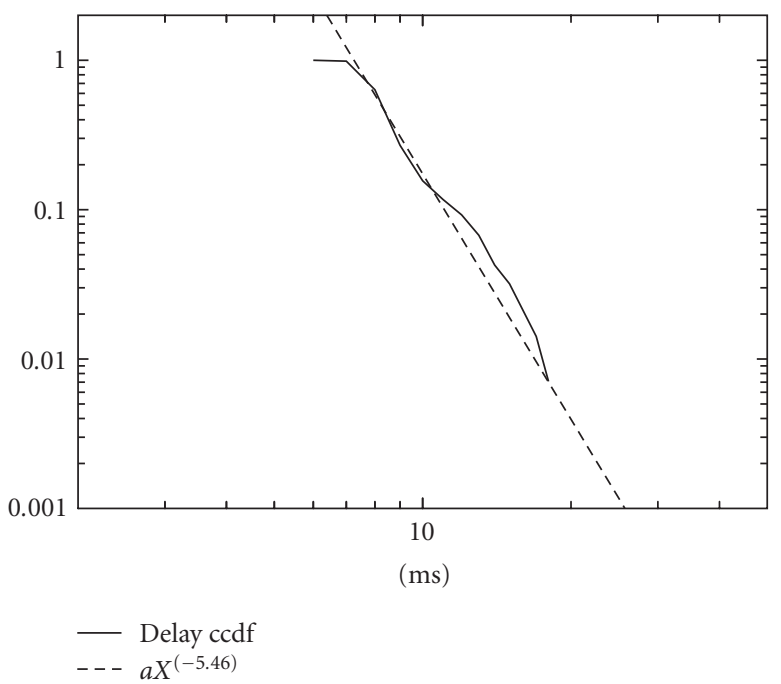

(a) Sending rate of $2 \mathrm{pkt} / \mathrm{s}$

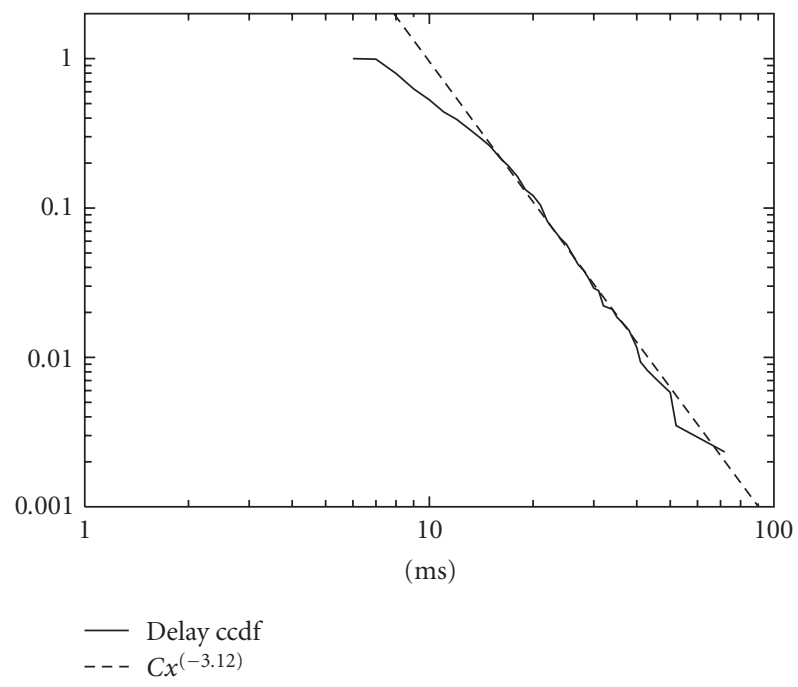

(b) Sending rate of $8 \mathrm{pkt} / \mathrm{s}$

FIgURE 9: Comparison between end-to-end delay distribution and the corresponding power law.

that the curves are shifted to larger delay values when the packet size increases. The shift in the delay distribution corresponds to the increase of the factor $c$ (route). In fact, bigger packets also require longer transmission times on the channel.

We note that, if the collision probability remains constant, according to (2), (7) bigger packets will only result in a shifted power law delay distribution with the same slope.

\section{Dependence of the delays on the routers}

We also measure the single-hop delay distributions along the path connecting the source to the destination. Let $W_{i}, i=$ $1 \cdots 5$, be the distribution generating function for each hop and $W$ the end-to-end delay distribution generating function. We compute the product $\prod_{i=1 \cdots 5} W_{i}$ and we compare it 


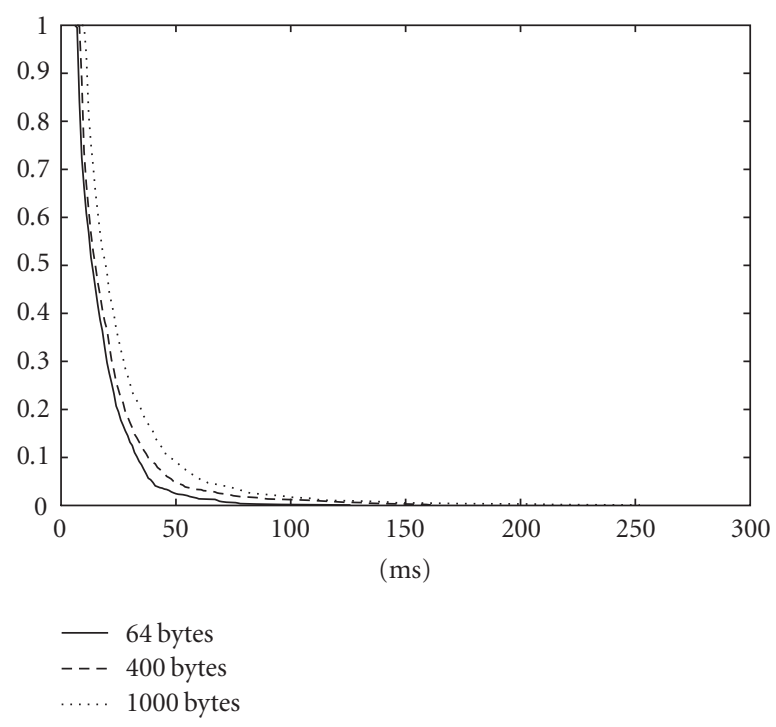

(a) Cumulative distribution function

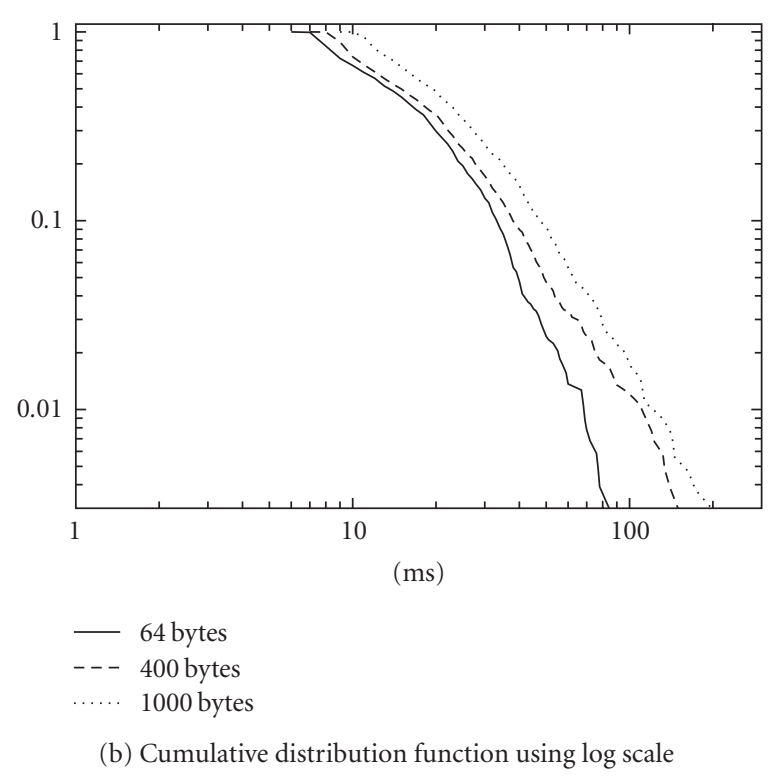

FIGURE 10: Impact of packet size on the delay distribution.

to $W$. In case the delays are independent within a route, the above product corresponds to the end-to-end delay distribution. As shown in Figure 11, the curves representing $W$ and $\prod_{i=1 \ldots 5} W_{i}$ are slightly different which means that there is a weak dependence between single-hop delays yet it is weaker when the network is lightly loaded. Notice that even when the independence assumption is not completely verified, the delay is still a power law as explained in Section 4.1.

\section{DELAY-DISTRIBUTION-BASED ROUTING}

As discussed earlier, routes with minimum average end-toend delay are not necessarily those which satisfy a delay constraint, defined generally as the probability $P(W>T)$ that

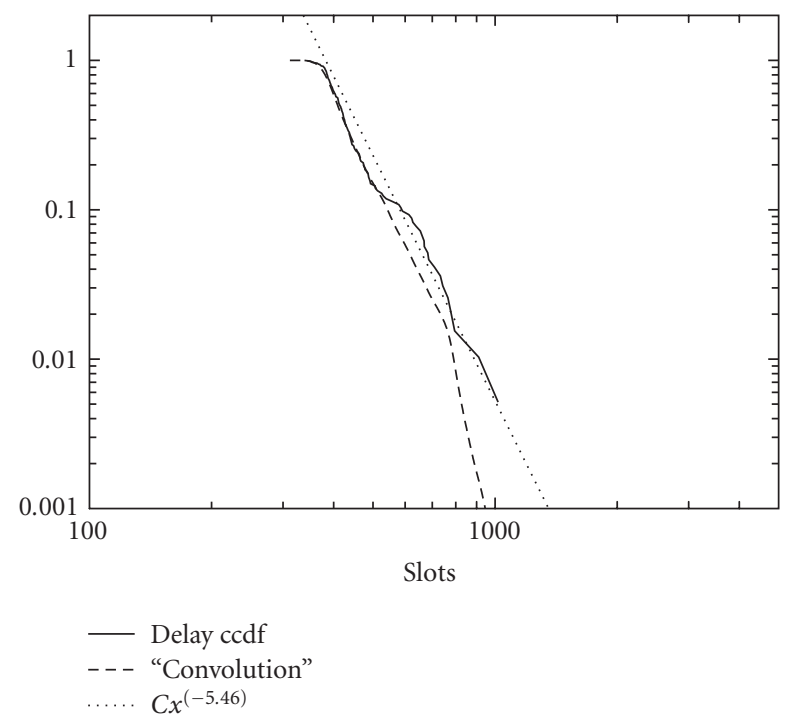

(a) Sending rate of $2 \mathrm{pkt} / \mathrm{s}$

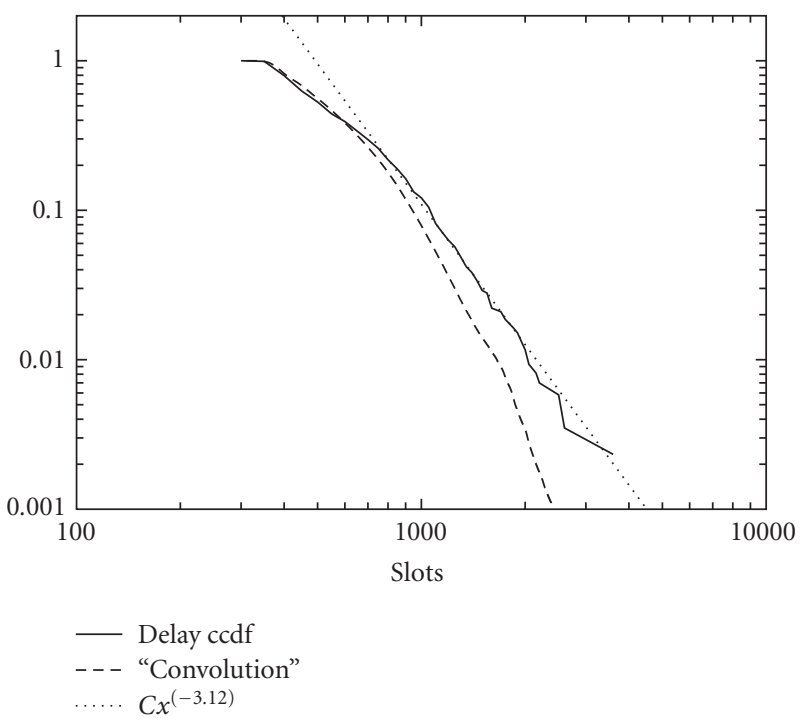

(b) Sending rate of $8 \mathrm{pkt} / \mathrm{s}$

FIGURE 11: Comparison between the end-to-end distribution and the convolution of single hop distributions.

the delay $W$ exceeds a maximum acceptable delay $T$, which is specified by the application layer. To compute such a probability, we need to know the delay distribution in every node, as presented in Section 5, instead of only the average delay. The problem of delay-distribution-based routing consists in finding a route that satisfies the application end-to-end delay requirement $P(W>T)<\epsilon$ for a given connection. Multiple routes satisfying such a delay constraint can be found in the network, hence a routing algorithm must select one among them. In this section, we explore two possible directions. The first direction consists in finding the optimal route that minimizes $P(W>T)$. The second direction consists in finding the shortest route (in hops) that satisfies the requirement $P(W>T) \leq \epsilon$. 


\section{Finding the optimal route}

In general, finding the optimal route with respect to a delay distribution is NP hard [7]. But if we stick to the asymptotic expression, we can find a polynomial Dijkstra-like algorithm. The problem is to find the route that provides the best asymptotic expansion of the quantity $P(W$ (route $)>T)$ when $T \rightarrow \infty$. By best asymptotic expansion we mean the one that provides asymptotically the lowest $P(W$ (route) $>T)$. Since we expect that $P(W$ (route) $>T)$ is asymptotically equivalent to $\sum_{i \in \text { route }} c_{i}^{*} T^{1-B_{i}}$ (cf. Section 4 ), the idea consists in minimizing the sum of the leading terms of the onehop delay distributions along the route. Hence, the routing algorithm is effectively a Dijkstra algorithm, where the weights on the links are $c^{*} T^{1-B}$. Parameters $c^{*}$ and $B$ are calculated according to the analysis in Section 4 . The weight of the route is the sum of the weights of the links, and the optimal route is the route that minimizes this sum.

When $T$ is finite, the sum of the weights on a route gives an approximation of the end-to-end delay distribution

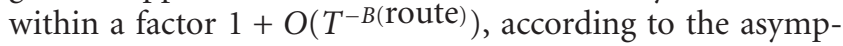
totic analysis. Since $B$ (route) $>1$, the algorithm is optimal within a factor $1+O\left(T^{-1}\right)$.

\section{Finding the shortest route satisfying the delay constraint}

As discussed previously, the shortest route that satisfies the constraint $P(W>T) \leq \epsilon$ is generally preferable to a much longer route that minimizes the quantity $P(W>T)$. Moreover, a major problem due to the use of any dynamic metric is route fluctuation. However, the proposed routing on the shortest path that verifies an over-delay ratio constraint provides more stable routes. In the previous section we described a polynomial search algorithm which is optimal within a factor $1+O\left(T^{-1}\right)$ hence for $T$ sufficiently large the search provides the optimal route. We showed that delay distribution routing can be reduced to polynomial routing based on an additive metric. In the present section we aim to find the shortest route according to a certain additive metric on links, that is, the number of hops, which satisfies a given constraint according to another additive metric, that is, the quantities $c^{*} T^{1-B}$.

In general such a multimetric optimization problem, also called multiconstrained path routing (MCP), is again NPhard. In [12], the authors present an overview of some proposed polynomial time approximation algorithms in the case of 2 metrics, and study the general case of $K \geq 2$ metrics. However, in our particular context, since the first metric can only take integer values, we can easily solve the problem in polynomial time with dynamic programming.

We model the network as a weighted graph. We consider a source node $s$. We denote by $v_{j}, j=1 \cdots n$, all the nodes in the network, where $v_{1}$ is the source $s$. Each link connecting two nodes $\left(v_{i}, v_{j}\right)$ is associated to a weight $w_{v_{i}, v_{j}}$ which corresponds to the asymptotic probability $c^{*} T^{1-B}$. For each node $v_{j}$ we define $p\left(i, v_{j}\right)$ as the smallest known value according to the second metric (the sum of the weights $w$ along the path) of all routes of length $i$ according to the first metric (hop count) that connect the source node $s$ to node $v_{j}$. Note that $i \leq n$, since the longest path in the network is at most $n$ hops.

We describe the algorithm as follows. We initialize all values $p\left(0, v_{j}\right)$, for $j=1 \cdots n$ to infinity except for $p\left(0, v_{1}\right)=0$. We will compute $p\left(i, v_{j}\right)$ for all $i, j$. For each $i \geq 1$ in increasing order, we compute the values $p\left(i, v_{j}\right), j=1 \cdots n$, using equation

$$
p\left(i, v_{j}\right)=\min _{c \in \mathcal{N}\left(v_{j}\right)}\left(p(i-1, c)+w_{c, v_{j}}\right)
$$

where $\mathcal{N}\left(v_{j}\right)$ is the neighborhood of node $v_{j}$ and $w_{c, v_{j}}$ is the weight of the link $\left(c, v_{j}\right)$. Notice that for all values of $i$ that are smaller than the distance between $s$ and $v_{j}$ we have $p\left(i, v_{j}\right)=$ $\infty$.

The aggregate computational cost of $p\left(i, v_{j}\right)$ for all nodes $v_{j}$ and for a given $i$ is $O(m)$, where $m$ is the total number of links in the network. Hence, in the worst case, the total time needed to construct the table $p\left(i, v_{j}\right)$ for all possible values $i, j=0 \cdots n$ is $O(m n)$. Once the table has been constructed, the shortest route to any destination $d$ satisfying the required delay constraint, corresponds to the route of minimum $i$ such that $p(i, d)<\epsilon$. In case the algorithm computes the route for one particular destination, the iteration on the route length $i$ can stop as soon as a feasible route is found.

\section{CONCLUSION AND PERSPECTIVES}

In this paper, we analyze the delay distribution in 802.11 multihop networks. We demonstrate that for large values of $T$ the cumulative delay distribution $P(W>T)$ is a power law. In practice, simulations show that this is true from $T$ equal to approximately twice the average. The delay distribution for a specific route can be derived based on MAC-layer as well as network-layer parameters, hence we present a cross-layer solution for a delay estimation protocol as an extension to the OLSR routing protocol. Furthermore, the information from this protocol can be used to compute the route that satisfies the QoS delay requirements specified by a multimedia application. In fact, delay-distribution-based routing is known to be an NP-hard problem. However, the asymptotic analysis in power law makes it possible to obtain a polynomial, Dijkstralike, algorithm.

It is important to note that the routing algorithm does not guarantee that the calculated route will satisfy the delay constraint after launching the new traffic. In case the new connection has a significant impact on the network conditions, it is necessary to dynamically control the delay, in order to check whether the constraint is still verified. Due to its proactive nature, the proposed delay estimation protocol allows to compute periodically the end-to-end delay, thus the routes can be readjusted. Therefore, it is interesting to combine the proposed delay routing algorithms with a mechanism providing dynamic delay control, as well as admission control when the connection delay requirements cannot be satisfied. In future work, we intend to implement such mechanisms, and subsequently to evaluate the performance of the proposed routing algorithms in this context.

Another direction for further research is to establish a link between bandwidth and delay requirements in the 
context of wireless multihop networks. This would permit an adaptation of bandwidth-QoS routing solutions for delaysensitive applications as well. Such a link can be established using the notion of equivalent bandwidth. In this particular case the equivalent bandwidth on a given path would correspond to the capacity of the network in transferring packets which satisfy this delay constraint. In [13], the authors show how to extend the effective bandwidth concept in order to provide a general model for a wireless channel in terms of connection level QoS metrics. It would be interesting to adapt the proposed model to the considered context of multihop IEEE 802.11 networks.

\section{APPENDIX}

Proof of Theorem 1. We fix $L$ and $p$ and set $e^{-\theta}=C(z)$ and denote $j(\theta, k)=\beta(z, k)$. We have $j(\theta, k)=\left(\left(1-e^{-k \theta}\right) /\right.$ $k \theta) f(\theta)(1-p+p j(\theta, 2 k))$, with $f(\theta)=e^{\theta}\left(\theta /\left(1-e^{-\theta}\right)\right) z^{L}$.

It is clear that $\theta=(1-z) C^{\prime}(1)+O\left((1-z)^{2}\right)$. We define $g(\theta)=\prod_{i \geq 1}\left(\left(1-e^{-\theta 2^{-i}}\right) / \theta 2^{-i}\right)$. Thus if

$$
\nu(\theta, k)=g(k \theta) j(\theta, k),
$$

then $v(\theta, k)=g(2 k \theta) f(\theta)(1-p)+p f(\theta) v(\theta, 2 k)$. And $v(\theta, k)=((1-p) / p) \sum_{i \geq 1}(f(\theta) p)^{i} g\left(2^{i} k \theta\right)$.

It can be proven that function $g(\theta)$ is analytical and behaves like $1+O(\theta)$ when $\theta \rightarrow 0$ and converges to zero faster than any power law when $\theta \rightarrow \infty$. Let $r_{B}(\theta)$ be the polynomial of degree $\lfloor B\rfloor$, which is the Taylor expansion of $g(\theta) e^{\theta}$ at $\theta=0$. Recall that $B=-\log _{2} p$.

Let $g_{B}(\theta)=g(\theta)-r_{B}(\theta) e^{-\theta}$. Clearly, $g_{B}(\theta)=O\left(\theta^{\lceil B\rceil}\right)$ when $\theta \rightarrow 0$. We have $v(\theta, k)=u_{B}(\theta)+((1-p) /$ p) $\sum_{i \geq 1}(f(\theta) p)^{i} g_{B}\left(2^{i} k \theta\right)$ with

$$
u_{B}(\theta)=\left(\frac{1-p}{p}\right) \sum_{i \geq 1}(f(\theta) p)^{i} r_{B}\left(2^{i} k \theta\right) e^{-2^{i} k \theta} .
$$

Clearly, $u_{B}(\theta)$ is an analytical function with $u_{B}(\theta)=1+$ $O(\theta)$. Let $\nu_{B}(\theta, k)=((1-p) / p) \sum_{i \geq 1}(f(\theta) p)^{i} g_{B}\left(2^{i} k \theta\right)$. We will show that $\mu_{B}(\theta, k)=\theta^{-B} \nu_{B}(\theta, k)$ is bounded when $\theta \rightarrow 0$. Let $h_{B}(\theta)=\theta^{-B} g_{B}(\theta)$. We have $\mu_{B}(\theta, k)=((1-$ $p) / p) \sum_{i \geq 1}(f(\theta))^{i} h_{B}\left(2^{i} k \theta\right) k^{B}$.

Since $f(\theta)=1+O(\theta)$, when $\theta \rightarrow 0$, we have $\mu_{B}(\theta, k)$ which converges to $\alpha(\log \theta)=\sum_{i} h_{B}\left(2^{i} k \theta\right) k^{B}$, the sum being on all integers $i$, including the negative integers. The sum converges because $h_{B}(\theta)=O\left(\theta^{\epsilon}\right)$ with $\epsilon=\lceil B\rceil-B$ and $h_{B}(\theta)$ decays faster than any power law. Notice that function $\alpha(x)$ is periodic of period $\log 2$.

Therefore $j(\theta, k)=v(\theta, k) / g(k \theta)$ has asymptotic expansion $u_{B}(\theta) / g(k \theta)+\alpha(\log \theta) \theta^{B}+O\left(\theta^{B+\epsilon}\right)$. The theorem follows with a change of variable.

Proof of Theorem 2. From the previous theorem it comes that $\beta(z)$ fits Flajolet-Odlyzko asymptotic conditions [9]. By writing the function $\alpha(\log (1-z))$ as a Fourier series $\alpha(\log (1-$ $z))=\sum_{n} \alpha_{n}(1-z)^{2 i n \pi / \log 2}$, and applying Flajolet-Odlyzko theorems, we have $P(S>T)=\left(W_{\min } C^{\prime}(1)\right)^{B} \alpha^{*}(\log T) T^{-B}+$ $O\left(T^{-B-1}\right)$, where $\alpha^{*}$ is periodic in $\log T$, of period $\log 2$ : $\alpha^{*}(\log (T))=\sum_{n}\left(\alpha_{n} / \Gamma(2-B-2 i n \pi / \log 2)\right) T^{2 i n \pi / \log 2}$.
Proof of Theorem 3. We substitute the expansion for $\beta(z)$ around $z=1$, derived in Theorem 1 , in the formula for $q(z)$ given by (6). The theorem follows by using the expansion around $z=1$ in equation $w(z)=q(z) \beta(z)$.

Proof of Theorem 4. We use the result of the previous theorem and we apply Flajolet-Odlyzko theorems on $w(z)$.

\section{ACKNOWLEDGMENT}

Part of this work was presented at the 1st Workshop on resource Allocation in Wireless Networks, April 3rd, 2005, Riva Del Garda, Italy.

\section{REFERENCES}

[1] IEEE 802.11 Standard, "Wireless Lan Medium Access Control and Physical layer Specifications," June 1997.

[2] T. Clausen and P. Jacquet, "Optimised Link State Routing (OLSR)," IETF RFC 3626.

[3] G. Bianchi, "Performance analysis of the IEEE 802.11 distributed coordination function," IEEE Journal on Selected Areas in Communcations, vol. 18, no. 3, pp. 535-547, 2000.

[4] H. Zhai and Y. Fang, "Performance of wireless LANs based on IEEE 802.11 MAC protocols," in Proceedings of the 14th IEEE on Personal, Indoor and Mobile Radio Communications (PIMRC '03), vol. 3, pp. 2586-2590, Beijing, China, September 2003.

[5] O. Tickoo and B. Sikdar, "Queueing analysis and delay mitigation in IEEE 802.11 random access MAC based wireless networks," in Proceedings of the 23d Annual Joint Conference of IEEE Computer and Communications Societies (INFOCOM '04), vol. 2, pp. 1404-1413, Hong Kong, March 2004.

[6] J. Chen and Y. Yang, "Multi-hop delay performance in wireless mesh networks," in Proceedings of IEEE Global Telecommunications Conference (Globecom '06), pp. 1-5, San Francisco, Calif, USA, November-December 2006.

[7] R. A. Guerin and A. Orda, "QoS routing in networks with inaccurate information: theory and algorithms," IEEE/ACM Transactions on Networking, vol. 7, no. 3, pp. 350-364, 1999.

[8] J. W. Cohen, "A heavy-traffic theorem for the GI/G/1 queue with a Pareto-type service time distribution," Journal of Applied Mathematics and Stochastic Analysis, vol. 11, no. 3, pp. 247-254, 1998.

[9] P. Flajolet and A. M. Odlyzko, "Singularity analysis of generating functions," SIAM Journal on Discrete Mathematics, vol. 3, no. 3, pp. 216-240, 1990.

[10] H. Badis, A. Munaretto, K. Al Aghal, and G. Pujolle, "Optimal path selection in a link state QoS routing protocol," in Proceedings of the 59th IEEE Vehicular Technology Conference (VTC '04), vol. 5, pp. 2570-2574, Milan, Italy, May 2004.

[11] K. Fall and K. Varadhan, The ns manual, 2002, http://www.isi .edu/nsnam/ns/ns-documentation.html.

[12] G. Xue, W. Zhang, J. Tang, and K. Thulasiraman, "Polynomial time approximation algorithms for multi-constrained QoS routing," to appear in IEEE/ACM Transactions on Networking.

[13] D. Wu and R. Negi, "Effective capacity: a wireless link model for support of quality of service," IEEE Transactions on Wireless Communications, vol. 2, no. 4, pp. 630-643, 2003. 

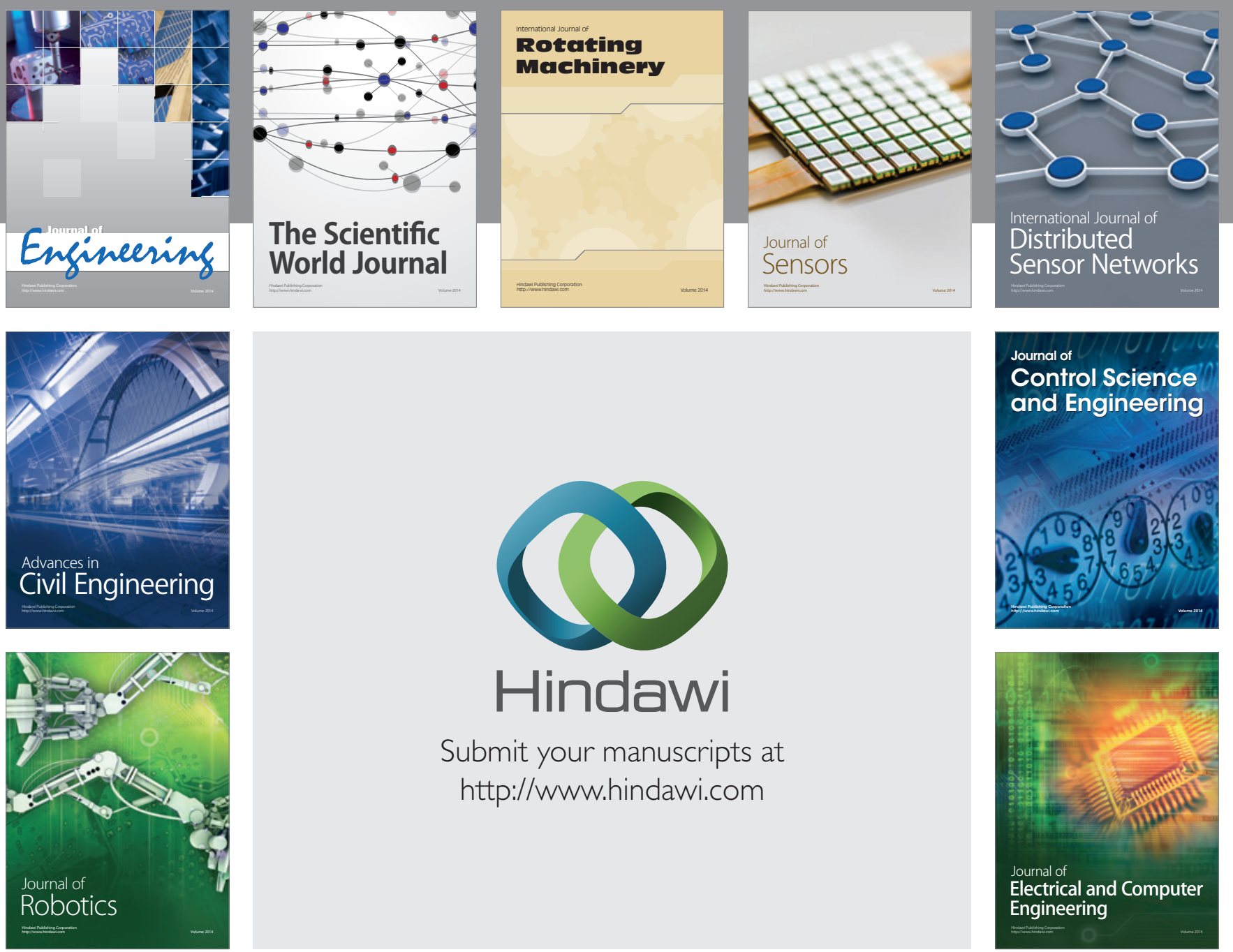

Submit your manuscripts at

http://www.hindawi.com
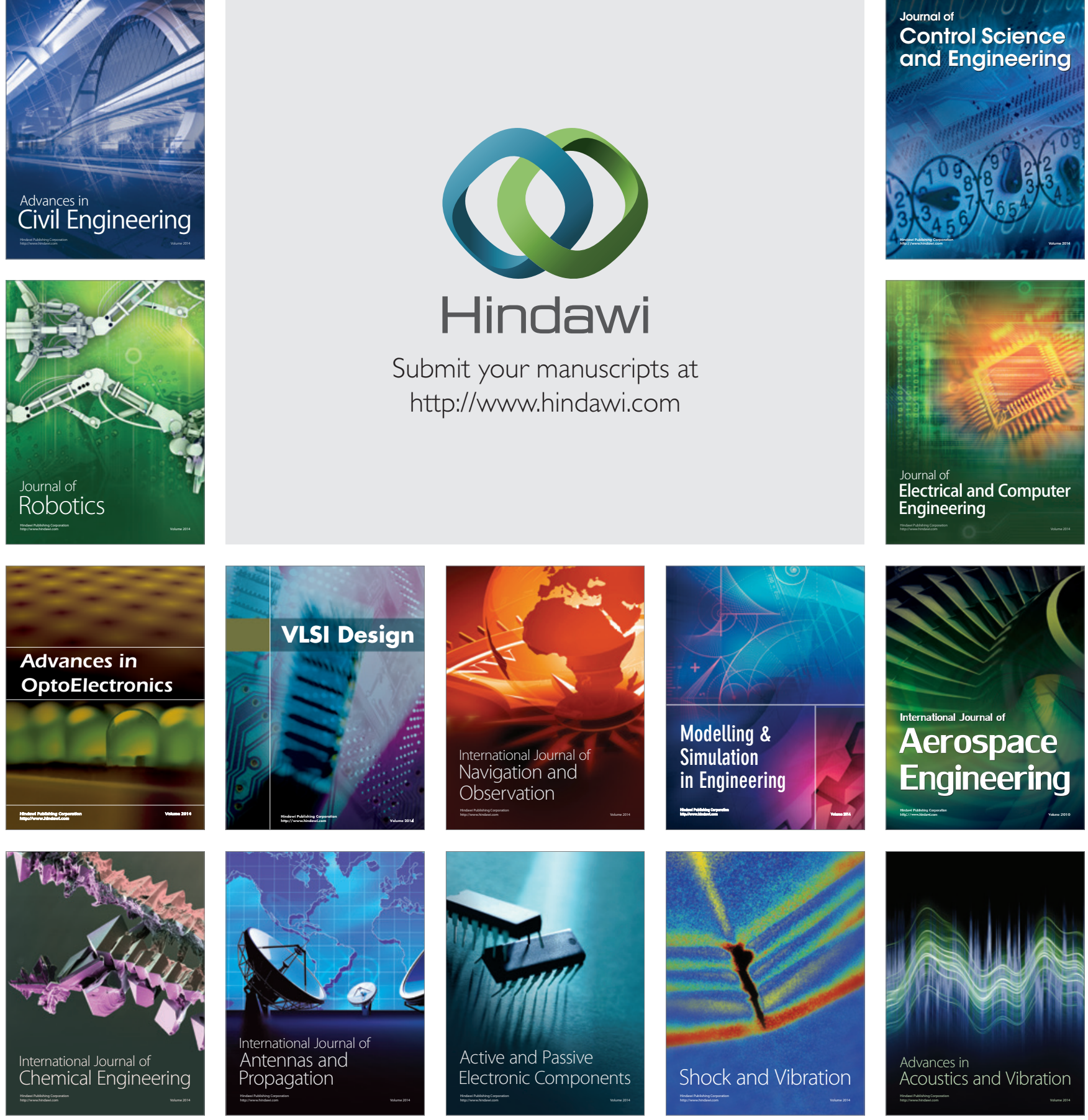